\title{
Real-time extraction of growth rates from rotating substrates during molecular-beam epitaxy
}

\author{
D. A. Collins, G. O. Papa, and T. C. McGill \\ T. J. Watson, Sr. Laboratory of Applied Physics, California Institute of Technology 128-95, Pasadena, \\ California 91125
}

(Received 14 March 1994; accepted 28 July 1995)

\begin{abstract}
We present a method for measuring molecular-beam epitaxy growth rates in near real-time on rotating substrates. This is done by digitizing a video image of the reflection high-energy electron diffraction screen, automatically tracking and measuring the specular spot width, and using numerical techniques to filter the resulting signal. The digitization and image and signal processing take approximately $0.4 \mathrm{~s}$ to accomplish, so this technique offers the molecular-beam epitaxy grower the ability to actively adjust growth times in order to deposit a desired layer thickness. The measurement has a demonstrated precision of approximately $2 \%$, which is sufficient to allow active control of epilayer thickness by counting monolayers as they are deposited. When postgrowth techniques, such as frequency domain analysis, are also used, the reflection high-energy electron diffraction measurement of layer thickness on rotating substrates improves to a precision of better than $1 \%$. Since all of the components in the system described are commercially available, duplication is straightforward. (c) 1995 American Vacuum Society.
\end{abstract}

\section{INTRODUCTION}

Reflection high-energy electron diffraction (RHEED) has long been used in molecular-beam epitaxy (MBE) to measure growth rates, since under the proper growth conditions, the intensity of the specular RHEED spot varies periodically with a frequency equal to the growth rate. ${ }^{1,2}$ To date, measurements of growth rates have been largely limited to stationary substrates, although Turner et al. have succeeded in extracting growth rates from rotating substrates using Fourier transform techniques. ${ }^{3}$ However, by its nature, this method is not real-time since several oscillations are needed to determine the growth rate. As a result, the previously reported technique cannot be used to actively control layer thicknesses but rather it is limited to determining the thicknesses of layers after they have been deposited. van der Wagt et al. demonstrated another technique for growth rate measurements on a rotating substrate. ${ }^{4}$ They used high speed substrate rotation $(\sim 400 \mathrm{rpm}$ for growth rates of $1 \mathrm{ml} / \mathrm{s})$ and a custom-built electrical filter to separate specular spot intensity variations due to rotation from those due to epilayer growth. They were successful in extracting the growth oscillations; however, the high speed rotation which the authors said led to excessive wear and the custom circuitry limited the application of their technique.

In this paper we present a method for measuring MBE growth rates in near real-time on rotating substrates by processing the data in the time domain rather than in the frequency domain without employing custom-built hardware while using substantially lower substrate rotation rates $(\sim 10$ rpm). This is accomplished by digitizing a video image of the RHEED screen, measuring the specular spot width, numerically filtering the resulting signal, and finally counting the oscillation peaks. The digitization and image and signal processing take approximately $0.4 \mathrm{~s}$ to accomplish, so this technique offers the MBE grower the ability to actively adjust growth times in order to achieve a desired layer thick- ness. The real-time growth rate measurement described below has a precision of approximately $2 \%$. When postgrowth techniques, such as the one previously mentioned, ${ }^{3}$ are included, the RHEED measurement of layer thickness on rotating substrates improves to a precision of better than $1 \%$.

\section{EXPERIMENT}

The epilayers used to obtain the data reported on here were grown in a Perkin-Elmer $430 \mathrm{MBE}$ chamber. All of the data shown here was obtained from GaAs layers grown on (100) surfaces at substrate temperatures of $600{ }^{\circ} \mathrm{C}$, as determined by an optical pyrometer calibrated to the GaAs oxide desorption point. Similar results were obtained from InAs, AlAs, $\mathrm{Al}_{x} \mathrm{Ga}_{[1-x]}, \mathrm{GaSb}$, and AlSb epilayers. Similar results were obtained from both In-bonded and In-free mounted substrates, although all of the data shown was acquired from In-bonded substrates. A Perkin-Elmer 20-330 RHEED gun was used to acquire the data using $\sim 0.25 \mathrm{~mA}$ of current and an angle of incidence of $\sim 1.5^{\circ}$. The energy of the RHEED electrons was varied from 7.5 to $10 \mathrm{keV}$ with no substantial change in the results.

To obtain the RHEED image, a video camera is focused on the diffraction pattern. The video signal is digitized onto a $640 \times 480$ array of single-byte data with a DataCell S2200 framegrabber installed in a SPARC 2 workstation. The digitized video image resides in memory located on the framegrabber card which can be directly accessed and manipulated via C-language pointers. As a result, the video image does not need to be copied into the computer's memory which greatly increases the framegrabbing rate. The system can digitize up to $60 \mathrm{frames} / \mathrm{s}$, depending on the amount of image processing being done, with the maximum framegrabbing rate selected by the operator. In addition, the look-up tables used by the analog to digital converters in the framegrabber card are software programmable. This allows the operator to process the video image as it is digitized, 
which does not contribute to the computational overhead. Using this feature of the framegrabber card, the operator can interactively remove constant backgrounds and adjust the contrast and brightness of the digitized image.

The extraction of usable information from the RHEED image of a rotating substrate can be broken into two parts: image processing which generates a usable stream of data, and signal processing which extracts information from that data stream. By their nature, RHEED screens produce images that are continuous in time. Practical considerations, however, force us to adopt a method that first captures the continuous time RHEED screen in a video frame stream with a characteristic frame rate ( $\sim 30$ fps for standard video equipment). From each such video frame we extract a single data point which means, assuming the image processing rate is sufficiently fast, that we obtain series of data points at a rate of $\sim 30 \mathrm{fps}$. Using the signal processing techniques discussed below, we extract the strongest frequency component of this data stream from a range surrounding the expected growth rate. We further demonstrate that this component does in fact correspond to the actual growth rate.

Our setup starts with a video camera directed at the RHEED screen providing video frames of the RHEED pattern. Since the specular spot occupies a relatively small percentage of the total image, the computer program defines a framing box that completely surrounds the specular spotwhich is assumed to be the brightest diffraction spot. The processing of video frames is then reduced to counting the number of pixels within this box whose intensity fall above a preselected threshold. However, when used with a rotating substrate, the specular spot moves within the video frame due to the changing diffraction conditions. To compensate, the program constantly recenters the framing box. The net result is that we obtain a pixel count that corresponds to the size of the specular spot for each successive video frame. With our setup this stream of pixel counts is not evenly spaced which leads us to linearly interpolate between data points. From this linear interpolation we obtain an evenly spaced (in time) series of measurements of the size of the specular spot which we represent as the discrete sample set $\left\{x_{i}, t_{i}\right\}$ where $\Delta t \equiv t_{i}-t_{i-1}$ is constant.

The set $\left\{x_{i}, t_{i}\right\}$ is a sample of a continuous process $x(t)$ that contains information about the actual material growth rate. For a stationary substrate this specular spot size process is closely correlated with the actual growth rate while for a rotating substrate, this process represents the growth rate modulated by the substrate rotation. In general, it is possible to select growth rates that differ substantially in frequency from the rotation rate thus allowing digital filtering techniques to be used to extract this growth rate component from the composite signal. By keeping only a range of frequencies around the growth rate the noise due to the substrate rotation is strongly suppressed. Using these digital filters it is possible to extract a reliable growth rate in just such a manner. Specifically, we select a range (say 0.8 to 1.2 monolayer per second for an expected 1 monolayer per second growth) centered on the growth rate, eliminate all frequency components outside of this range, and count peaks to obtain the measured growth rate. We implicitly assume that the strongest compo-

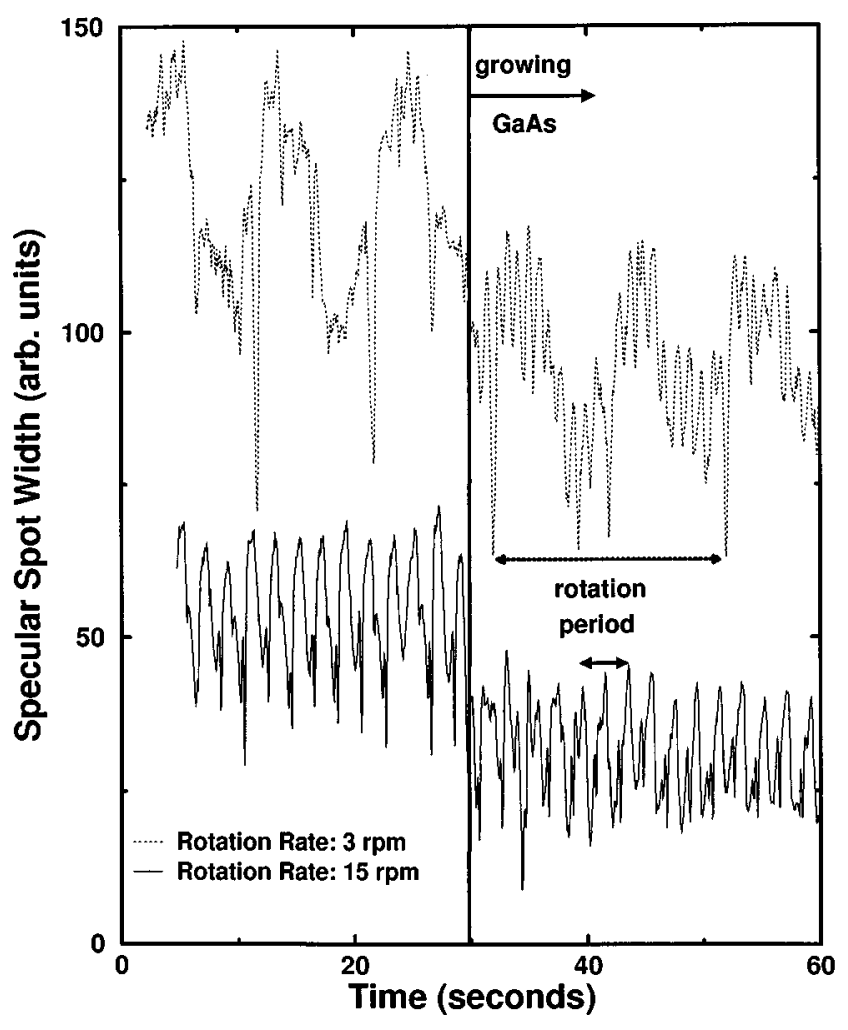

FIG. 1. Plots of the specular spot width automatically measured on a rotating substrate. The solid vertical line marks the start of the growth and the two horizontal lines show the rotation period. The data have been vertically offset for clarity.

nent in this range represents the actual growth. This is verified by comparing the growth rate extracted from a rotating substrate with that obtained from a stationary one under the same growth conditions.

Digital filtering techniques are used so that this growth information can be obtained in something like real-time. The time lag between the filter output $y_{j}$ and the leading edge of the data stream $x_{i}$ can be selectively controlled, however, a lag of $\sim 0.3 \mathrm{~s}$ is necessary to obtain good results. To illustrate, consider the actual recursive digital filtering equation:

$$
y_{k}=\sum_{n=0}^{L} b_{n} x_{k-n}-\sum_{n=1}^{L} a_{n} y_{k-n}
$$

where $y_{k}$ depends on $\left\{x_{k}, \ldots, x_{k-L}\right\}$ as well as $\left\{y_{k-1}, \ldots, y_{k-L}\right\}$. Typically, $L$ is on the order of 10 while the stream of $x_{i}$ 's are arriving at a rate of approximately 30 per second thus the $0.3 \mathrm{~s} \mathrm{lag}$. The selection of the $a_{n}$ 's and $b_{n}$ 's in Eq. (1) constitute the filter design problem and it is this selection that controls the passband or stop-band characteristics of the filter. This is a well studied problem and software is available off the shelf to accomplish the filter design. ${ }^{5}$ For this particular problem, infinite impulse response filters are used (fifth order-40 $\mathrm{dB}$ stop-band rejection-3 $\mathrm{dB}$ ripple). This form of digital filtering requires a relatively small number of coefficients, thus achieving the short lag times necessary to enable use of this technique as an active growth control feedback mechanism. The type of 


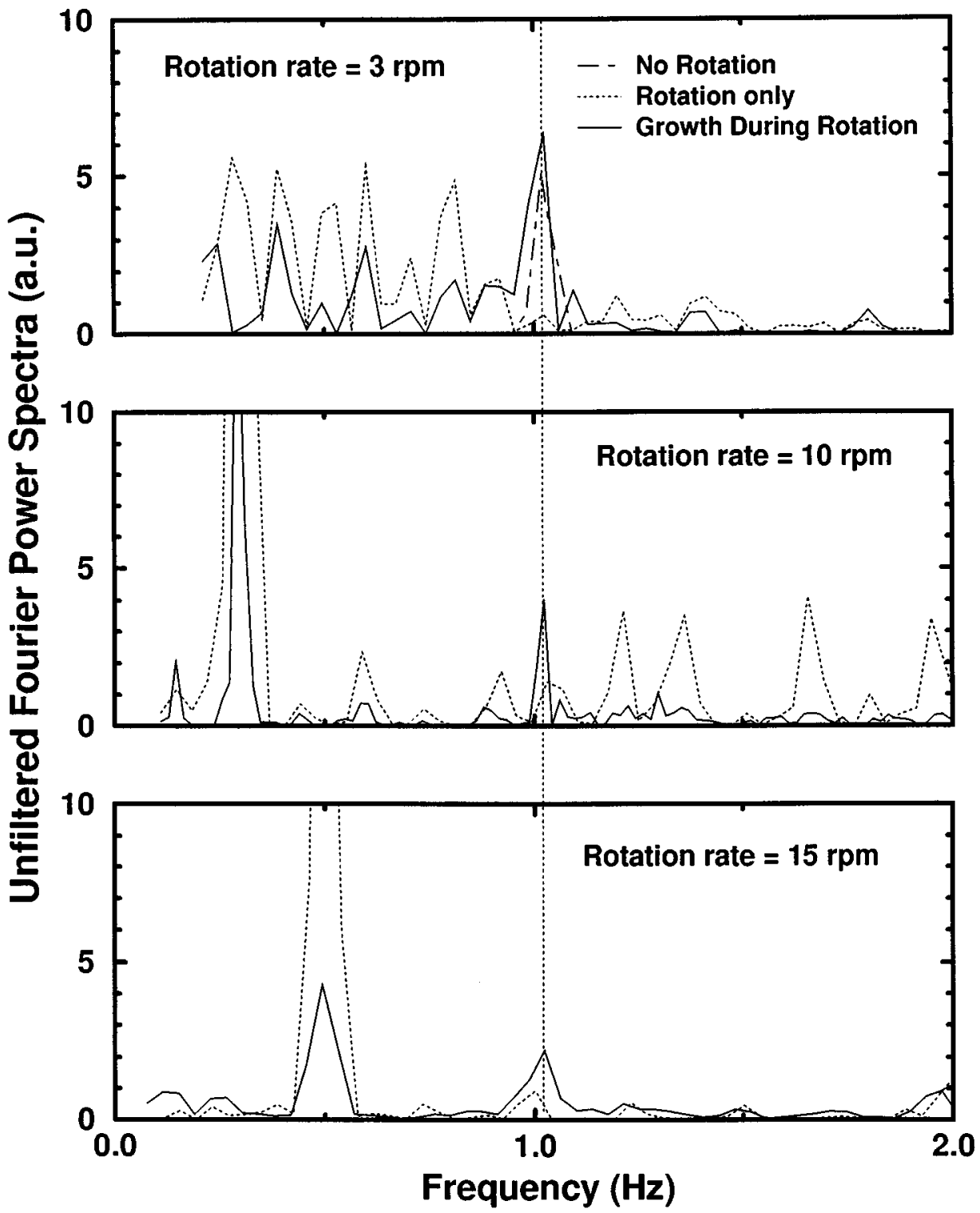

FIG. 2. Plots of the Fourier power spectra of the dynamic specular spot width during rotation. In each of the panels, the dotted line is the spectra of rotation only, while the solid line is for growth during rotation. The dashed line in the upper panel is the spectrum of RHEED oscillations taken from a stationary substrate. The dotted vertical line marks the growth rate obtained from a stationary substrate.

filter-Chebyshev type I or II or Butterworth-whether it is a bandpass or bandstop filter, the number and order of filter sections desired and the attenuation are all chosen by the operator. Once the operator has selected the filter characteristics, the $a_{n}$ 's and $b_{n}$ 's in Eq. (1) are calculated (this typically requires less than $1 \mathrm{~s}$ of computation).

In practice, the filter's characteristics are chosen by examining the Fourier power spectrum of the measured dynamic spot width during rotation without growth, keeping the expected growth rate in mind. A similar approach has been implemented using custom-built hardware. ${ }^{4}$ However, a software based method is vastly superior in its flexibility since it allows the MBE operator to tailor the filter for a particular rotation and growth rate. In addition, with a sophisticated numerical filtering technique the software approach is easily fast enough to allow real-time data collection.

The peak-counting routine counts zero crossings of the numerical derivative of either the smoothed or filtered signal. The derivative is determined by taking the dot product between a vector of data points and a vector of appropriately chosen weights. ${ }^{6}$ Since this method only involves multiplication and addition, it is not computer intensive. The derivative is determined from five data points which typically introduces a delay of roughly $0.15 \mathrm{~s}$ into the peak-counting routine-the computation itself is essentially instantaneous on the time scales of importance. The overall delay in the signal caused by the framegrabbing, filtering, and peak counting is approximately $0.33 \mathrm{~s}$. While this does not affect the growth rate fed into the MBE control loop, it must be accounted for in applications where layer growth times are determined by counting monolayers as they are deposited.

After growth, the entire RHEED signal can be analyzed to refine the real-time data. Two types of analysis have currently been implemented: Fourier transforms and binning of 


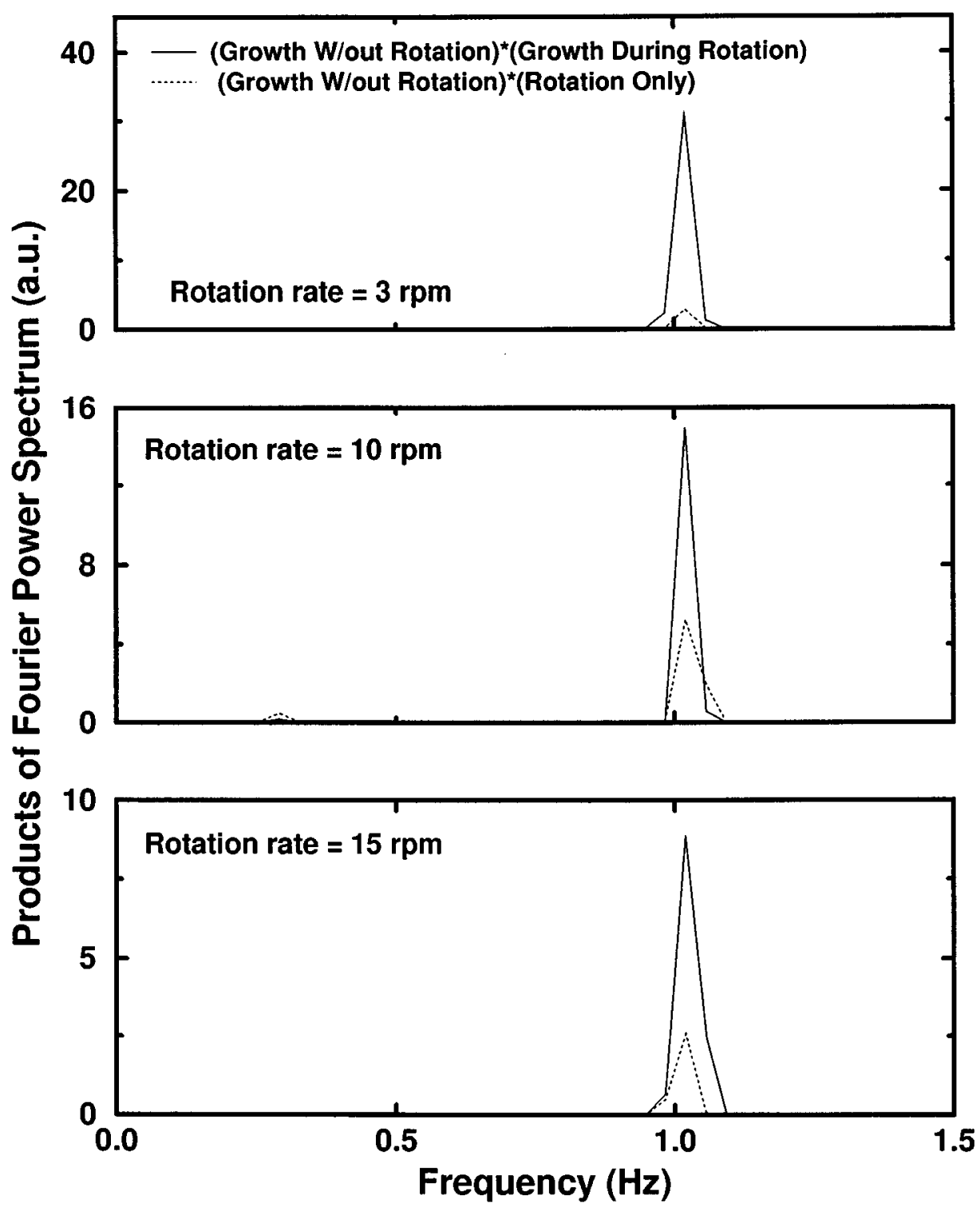

FIG. 3. Product of the power spectrum of growth on a stationary substrate with the power spectra obtained from a rotating substrate with and without growth of an epilayer. In each of the panels the solid line is the product of the spectrum without rotation, with spectra during rotation with growth. The dotted line is the product of the spectrum without rotation and the spectra during rotation without growth.

the oscillation periods. Growth rates determined from stationary substrates are not affected by the postgrowth analysis. The precision of growth rates extracted from rotating substrates increases from approximately $2 \%$ to better than $1 \%$ with postgrowth analysis, as will be shown later.

\section{RESULTS}

Obtaining growth rates from stationary substrates is straightforward. After initiating growth, the program automatically finds the specular RHEED spot and tracks changes in its size. The data stream is smoothed using a SavitzkyGolay algorithm ${ }^{6}$ after which a peak-counting routine identifies the times at which oscillation peaks occur and displays the measured growth rate in near real-time on the computer screen. Postgrowth techniques such as Fourier analysis or identification and subsequent rejection of extraneous oscillation peaks can be done to improve the precision of the mea- surement. Different measurements of growth rates on stationary substrates typically agree with each other to better than $1 \%$ without postgrowth analysis.

Extracting growth rates from rotating substrates is substantially more difficult since the variations in the specular spot intensity due to the changing diffraction conditions must be accounted for. Additionally, the necessity for real-time information severely restricts the numerical techniques available for the data analysis. In Fig. 1 we show the measured specular spot size during rotation for two different rotation frequencies. The data have been vertically offset for clarity, with the upper, dotted curve corresponding to a rotation rate of approximately $3 \mathrm{rpm}$, while in the lower, solid curve, the rotation rate is roughly $15 \mathrm{rpm}$. The solid vertical line marks the start of the growth, and the two horizontal lines show the rotation period. Two important points are clearly demonstrated. First, there is an obvious change in the signal when 


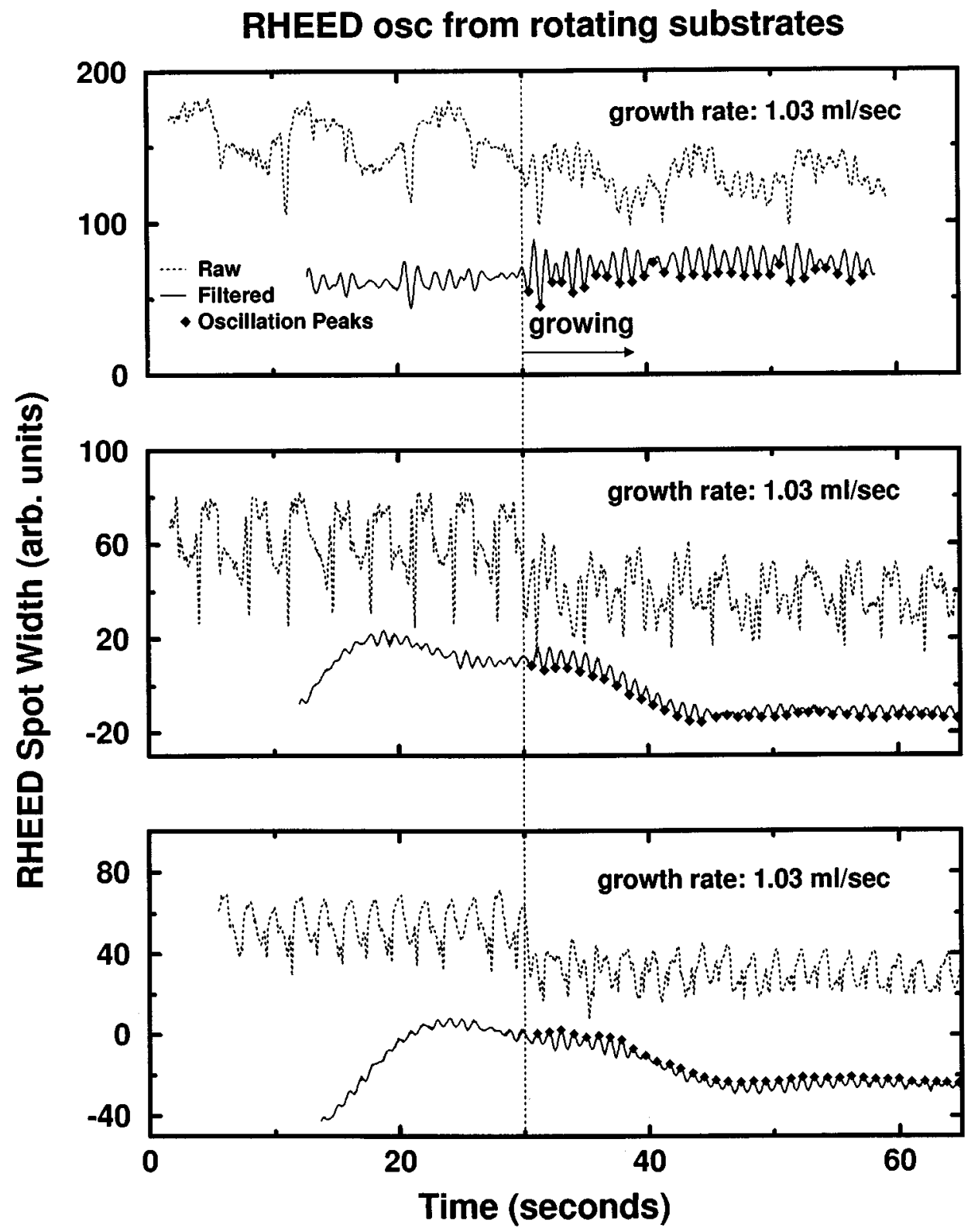

Fig. 4. Plots of the measured specular spot width during rotation with and without growth. In each of the panels, the upper dotted curve is the raw data, while the lower solid curve is the data after numerical filtering. The solid diamonds superimposed on the filtered data mark the positions of the oscillation peaks as determined by the peak-counting routine. Note that in the upper two panels the diamonds occur at local maxima, while in the bottom panel the diamonds are at local minima. Different peak-counting routines which trigger on the maxima for the upper two panels and on the minima for the lower panel were used. The dotted vertical line marks the time when the growth was started.

the growth is started. Second, the large changes in the signal due solely to the rotation must be accounted for when trying to measure oscillations due to the growth of the epilayer.

In Fig. 2 we plot the Fourier power spectra of the dynamic specular spot width during rotation. The upper, middle, and low panels correspond to rotation rates of approximately 3 , 10 , and $15 \mathrm{rpm}$, respectively. In each of the panels, the dotted line is the spectra of rotation only (RO), while the solid line is for growth during rotation (GDR). The dashed line in the upper panel is the spectrum of RHEED oscillations taken from a stationary substrate immediately prior to acquiring the data from rotating substrates. The dashed vertical line marks the growth rate obtained from the stationary substrate. The data in Fig. 2 demonstrate three important points. First, comparison of the spectra with and without growth, solid, and dotted lines, respectively, clearly demonstrates a strong enhancement of the Fourier component corresponding to the growth rate. Second, in each of the panels there is a Fourier component at the growth rate in the spectrum of data from RO. This component can be somewhat attenuated by adjusting the position of the substrate holder or by using substrates that are more closely aligned to a major crystal axis. In practice this is not necessary since subsequent filtering of the signal can effectively eliminate the uncertainty of this contribution. The important point is the clear enhancement of the Fourier component at the growth rate during GDR. Third, comparison of the power spectra of RO and GDR shows that the growth suppresses all of the observed Fourier compo- 


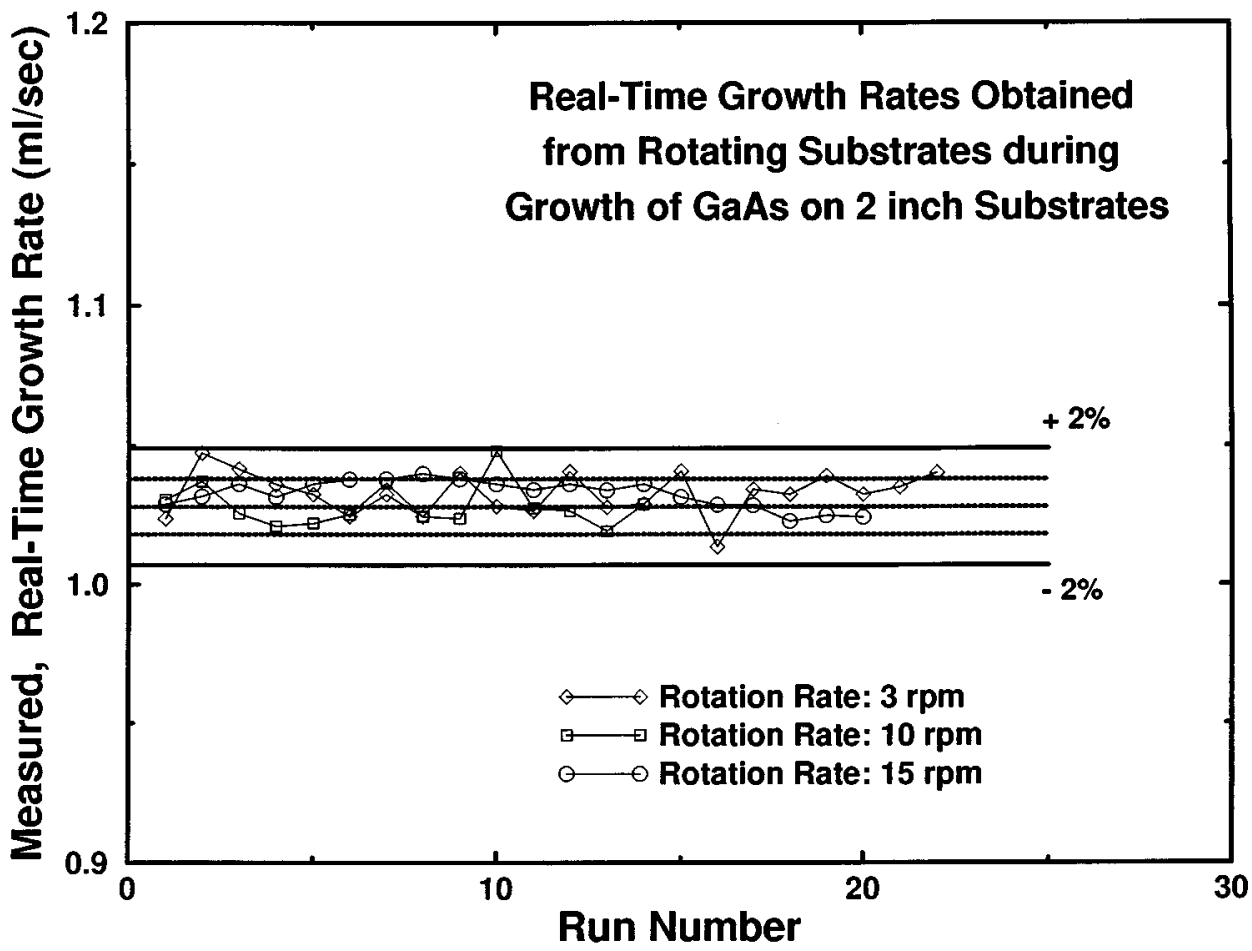

FIG. 5. Real-time growth rates measured on rotating substrates. The growth rates were measured consecutively while the temperature of the Ga oven was held constant. Growth interrupts of between 1 and 3 min were employed between measurements to ensure a smooth starting surface. The figure shows the measured real-time growth rates for substrates rotating at 3,10, and $15 \mathrm{rpm}$ (diamonds, squares, and circles, respectively).

nents. This is presumably due to the fact that RHEED patterns during growth are less sharp than during interrupts. This diffusing of the diffraction pattern tends to lessen the impact of changes in the diffraction conditions on the specular spot intensity, and hence the contribution to the Fourier amplitude at the growth frequency due solely to substrate rotation.

To more clearly show the enhancement of the Fourier component corresponding to the growth rate, in Fig. 3 we show the product of the power spectrum of growth on a stationary substrate with the power spectra obtained from a rotating substrate with and without growth of an epilayer. The upper, middle, and lower panels correspond to rotation rates of approximately 3,10 , and $15 \mathrm{rpm}$, respectively. In each of the panels the solid line is the product of the spectrum without rotation and the spectra of GDR. The dotted line is the product of the spectrum without rotation and the spectra during RO. The data in all of the panels conclusively show the enhancement of the Fourier component at the expected growth rate. This is especially noteworthy in light of the previously mentioned suppression of all Fourier components during growth.

It is important to note that the intensity scales on the panels in Fig. 3 are different. While the frequency component at the growth rate is enhanced during growth in each of the data sets, its absolute magnitude decreases as the rotation frequency increases. This is characteristic of all the data studied, and is possibly due to the finite sampling rate. However, the ratio of the Fourier components at the growth rate for GDR and during RO is not a monotonic function of the rotation rate. This ratio is approximately $12.9,2.9$, and 3.4 for rotation frequencies of 3,10 , and $15 \mathrm{rpm}$, respectively. The implications of these observations are twofold. First, better growth rate data is obtainable when using slower rotation rates when the rotation rate is of the same order as the growth rate. In practice, the minimum rotation rate is determined by the requirement that the substrate complete several rotations during the growth of the thinnest epilayer of interest, to ensure good thickness uniformity. Second, in order to optimize the extraction of growth rates, it is necessary to examine the Fourier spectra during RO. This allows the grower to choose a rotation rate that interferes the least with the expected oscillation signal due to growth. The optimal rotation rate must be determined for each growth rate of interest and is highly system dependent. In contrast, we have seen very little variation between different substrates, and the optimal rotation rate does not change after system vents.

The above discussion demonstrates that RHEED oscillations due to growth of an epilayer can be obtained from a rotating substrate. It is only necessary to filter the signal using the numerical techniques discussed above. Figure 4 shows plots of the measured specular spot width during rotation with and without growth. In each of the panels, the upper dotted curve is the raw data, while the lower solid curve is the data after numerical filtering. The upper, middle, and lower panels correspond to rotation rates of approximately 3, 10, and $15 \mathrm{rpm}$, respectively. The solid diamonds superimposed on the filtered data mark the positions of the oscillation peaks as determined by the peak-counting routine. The dotted vertical line marks the time when the growth was 
started. As can be seen from the data, the program can quite easily extract the growth oscillations from the filtered signal. The oscillations in the filtered signal before growth commences are due to Fourier components in the rotation near the growth rate. These can be rejected by either having the program monitor the opening and closing of the oven shutters, and only counting peaks during epilayer deposition, or by the operator signaling the start of growth.

To demonstrate the efficacy of the overall approach, we plot the results of a series of experiments where growth rates were measured on a rotating substrate in Fig. 5. The growth rates were measured consecutively while the temperature of the Ga oven was held constant. Growth interruptions of between 1 and 3 min were employed between measurements to ensure a smooth starting surface. The figure shows the measured real-time growth rates for substrates rotating at 3, 10, and $15 \mathrm{rpm}$ (diamonds, squares, and circles, respectively). Growth rates were measured with the substrate stationary before and after taking the data with rotation to ensure that the growth rate remained constant. As the data in Fig. 5 shows, the precision of the measurement is approximately $\pm 2 \%$ over almost 60 separate measurements. The growth rates displayed are the final growth rates determined by realtime extraction of RHEED oscillations due to epilayer growth. The period of the RHEED oscillations is determined and displayed in real-time. When postgrowth analysis is included, the precision of the measurement is better than $1 \%$. This shows that this technique can be used to either count the monolayers of a thin layer as they are deposited, allowing for active control of its thickness, or to determine a layer's thickness after growth.

The technique described above has an obvious, immediate application to MBE grown structures. Since the RHEED oscillations can be extracted in near real-time, active control of the thicknesses of very thin layers is possible. This should lead to improvements in run to run uniformity as well as allowing for interactive adjustment of layer thicknesses during the growth of structures such as Bragg reflectors. In addition, this control system is reasonably inexpensive since most MBE chambers are already equipped with RHEED sys- tems, and low cost 486-based desktop computers are easily fast enough to perform the computations described. An added benefit of a 486-based implementation of these algorithms is the wider variety of framegrabber cards available as compared to workstations. This broader range of product choices should give better performance at lower costs.

\section{SUMMARY}

In summary, we have presented a method for measuring MBE growth rates in near real-time on rotating substrates. This is accomplished by digitizing a video image of the RHEED screen, automatically tracking and measuring the specular spot width, and using numerical techniques to filter the resulting signal. The digitization and image and signal processing take approximately $0.4 \mathrm{~s}$ to accomplish, so this technique offers the MBE grower the ability to actively adjust growth times in order to achieve a desired layer thickness. The measurement has a demonstrated precision of approximately $2 \%$. This is sufficient to allow active control of epilayer thickness by counting monolayers as they are deposited. When postgrowth techniques, such as frequency domain analysis, are also used, the RHEED measurement of layer thickness on rotating substrates improves to a precision of better than $1 \%$.

\section{ACKNOWLEDGMENTS}

The authors wish to thank H. J. Levy for his helpful input on numerical signal processing techniques. This work was supported by the office of Naval Research under Contract No. N00014-93-1-0710.

${ }^{1}$ J. M. VanHove, C. S. Lent, P. R. Pukite, and P. I. Cohen, J. Vac. Sci. Technol. B 1, 741 (1983).

${ }^{2}$ J. H. Neave, B. A. Joyce, P. J. Dobson, and N. Norton, Appl. Phys. A 31, (1983).

${ }^{3}$ G. W. Turner and A. J. Isles, J. Vac. Sci. Technol. B 10, 1784 (1992)

${ }^{4}$ J. P. A. van der Wagt and J. S. Harris, presented at the North American Molecular-Beam Epitaxy Conference, Sept. 13-15, 1993, Stanford, CA.

${ }^{5}$ Signal Processing Algorithms in FORTRAN and c, edited by S. D. Stearns and R. A. David (Prentice-Hall, Englewood Cliffs, NJ, 1993).

${ }^{6}$ A. Savitzky and M. J. E. Golay, Anal. Chem. 36, 1627 (1964). 